\title{
Research on Roller Status Diagnosis of CRDM Based on SWT and HHT
}

\author{
Liming Zhang ${ }^{1}$, Lei Rong ${ }^{1}$, Meng Jiao ${ }^{1 *}$, Ling $\mathrm{Li}^{2}$ and Yaohua $\mathrm{Yu}^{3}$ \\ ${ }^{1}$ Department of Nuclear Energy Science and Engineering, Naval University of Engineering, Wuhan, Hubei, 430033, China \\ ${ }^{2}$ Wuchang University of Technology, Wuhan, Hubei, 430223, China \\ ${ }^{3}$ Shanghai First Machine Tool Works Co, Ltd, Shanghai, 200070, China
}

\begin{abstract}
For the control rod drive mechanism roller vibration signal's characteristics of nonlinear and nonstationary. Based on actual equipment life experiment and roller fault experiment, a status diagnosis method of roller of control rod drive mechanism based on Semi-soft wavelet threshold and Hilbert-Huang transform is proposed. Firstly, semi-soft wavelet threshold method is used to reduce noise interference and the influence of end-point effect on empirical mode decomposition, and the improved Hilbert transform method is used to extract the fault characteristics of roller vibration signal. The experimental results show that the method can effectively eliminate the interference of noises and realize the status diagnosis of the roller.
\end{abstract}

\section{Introduction}

As the key part of nuclear reactor, the technical condition of the control rod drive mechanism (CRDM) directly influences the safe operation of nuclear reactor. The sealed reluctance motor CRDM of a PWR realizes the reactor power regulation and operation by the roller parts of the rotor and the screw connecting the control rod to compose transmission gear to drive the control rod assembly to move up and down.1 The experience shows that as the main moving parts of the CRDM, the roller cannot avoid the fault mode of abrasion and bump with the increasing of operation time. When this fault mode achieves a certain degree or a serious fault appears, the CRDM cannot operate, which will have serious impact on the safety and operation of the reactor. Moreover, the roller works inside the main coolant pressure boundary of the primary system. Once the roller needs to be overhauled or to be replaced, the pressure boundary of the reactor must be cut, which will be involved in a big project needing a long maintain time. Therefore, monitoring and estimating the roller state in time and putting forward a reasonable maintaining or replacing advice combined with reactor refueling plan will have important practical significance.

Through CRDM life test research, it is found that due to environmental noise, internal structure noise, data acquisition system interference and complex signal transmission path and other reasons, the collected roller vibration signals have nonlinear and non-stationary characteristics. How to deal with such signals is the key to CRDM roller state assessment. Hilbert - Huang Transform(HHT) is a kind of adaptive time-frequency decomposition method[2-3], compared with other signal processing methods such as short-time Fourier Transform requirements according to the high frequency window function4, choose suitable Wigner distribution will produce the phenomenon such as secondary aliasing and difficult to choose appropriate kernel function processing Cohen, wavelet Transform is not adaptive characteristics5, its adaptive ability is strong, can better deal with the nonlinear and non-stationary signal6. HHT includes empirical mode decomposition (EMD) and Hilbert transform. EMD can adaptively decompose the signal into the sum of several intrinsic mode functions (IMF), and apply Hilbert transform to each IMF component to obtain the instantaneous amplitude and instantaneous frequency of the signal, thus obtaining the Hilbert three dimensions time-spectrum of the original signal7. The adaptive feature of HHT makes it can be used widely, however, there are several disadvantages in HHT, such as overenvelope, underenvelope and end effect[8-9]. Wavelet semi-soft threshold can effectively remove noise and reduce the influence of end-point effect on EMD. In this paper, the method of combining wavelet semi-soft threshold with improved HHT is adopted to extract the fault characteristics of vibration signal and realize the accurate judgment of roller status.

\section{Roller vibration signal acquisition}

The sealed reluctance motor CRDM is a fully sealed system, which is composed of stator, split rotor and pressure-resisting shell. The split rotor and roller are located inside the primary pressure boundary (as shown in Figure. 1). Therefore, the vibration sensor can only be arranged in the part outside the driving mechanism that is sensitive to the roller signal (as shown in figure 2). The vibration signal of the roller can be transmitted to the sensor through multiple transmission surfaces. The test

"Corresponding author's e-mail: nue_hexueyuan@sina.com 
bench is a full-life thermal test bench with sealed reluctance motor drive mechanism. The operating pressure and temperature of the bench are the same as the actual reactor. The signal acquisition device is a Danish B\&K3560 vibration tester. The sensor is installed on the outside of the pressure-resistant housing near the roller, and the sensor is fixed by welding the transfer block on

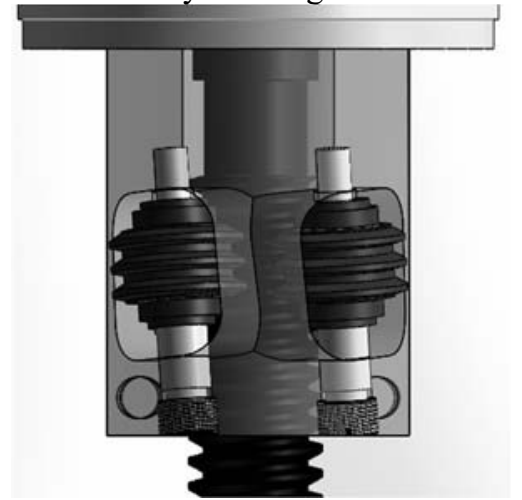

Figure 1. Diagram of rollers and lead screw.

After the life test is completed, based on the fact that the driving mechanism roller is likely to slip and bump with the lead screw in the actual use, the "groove" similar to the roller fault in the actual use is processed on the contact surface of the roller (as shown in Figure 3), and then the test is carried out on the thermal test bench and the vibration signal is collected.

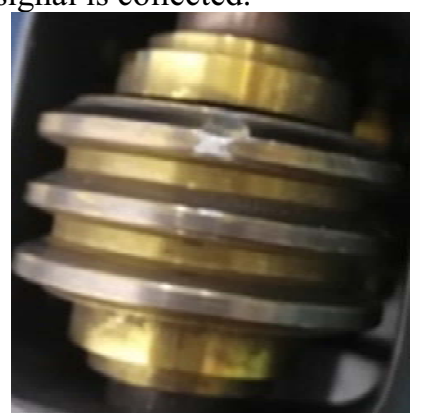

Figure 3. Defective diagram of roller.

\section{Feature extraction method of roller vibration signal}

HHT has a good performance in dealing with nonlinear and non-stationary time domain signals. Through EMD, complex vibration signals can be decomposed into IMF with physical significance. Through Hilbert transform, time spectrum of different IMF components can be obtained, and the instantaneous frequency contained in vibration signals in different frequency domains can be conveniently observed.

\subsection{Fault diagnosis method based on SWT}

The EMD method has the characteristics of adaptive, but when the collected vibration signal contains noise, the noise will participate in the decomposition, confuse the waveform characteristics of original signal, leading to the IMF component is inaccurate, therefore, must be conducted before the EMD decomposition to the signal the housing, the sampling frequency is $16384 \mathrm{~Hz}$. In the test process, the vibration signal is measured once every up and down cycle of the lead screw. The vibration signal acquisition covers the whole process of verifying the design life of the drive mechanism.

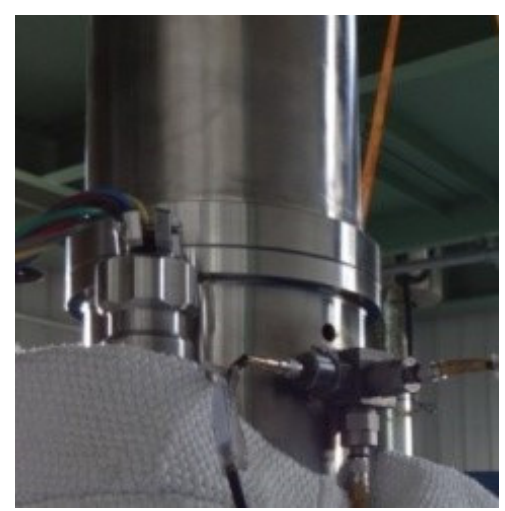

Figure 2. Schematic diagram of experimental bench.

noise reduction processing, the wavelet semi-soft threshold de-noising in the study of the vibration signal denoising of CRDM effect is good10.

\subsection{Empirical mode decomposition and Hilbert transform}

Empirical mode decomposition (EMD) is a method that decomposes the non-stationary and nonlinear signal to a set of stationary and linear data sequence. The decomposed data sequence set is the IMF, which satisfies the conditions: (1) the numbers of the extreme point and zero crossing point must be equal or differ by one; (2) the mean value of the envelope line constituted by the local maximum value on any point and the envelope line constituted by minimum value is zero.

For any signal $x(t)$, EMD decomposing procedures are as following:

1) Confirm all the local extreme points of $x(t)$, and use the cubic spline to connect all the local maximum value points to take shape of upper envelope line, then connect all the local minimum value points to take shape of lower envelope line. The upper and lower envelope lines should envelope all the data points.

2) Calculate the mean value of the upper and lower envelope lines: $m_{j}$.

$$
h_{j}=m_{j}-x(t)
$$

3) If $h_{j}$ satisfies the conditions of IMF, $h_{j}$ is the first component of $x(t)$; otherwise, set $h_{j}$ as the original data to repeat the procedure (1) and (2) so as to get $m_{j i}$, the mean value of the upper and lower envelope lines. Continually estimate if $h_{j i}=h_{j}-m_{j i}$ satisfies the conditions of IMF, if not, repeat the above circulation $\mathrm{n}$ times until $h_{j(n-1)-} m_{j n}=h_{j k}$ and $h_{j k}$ satisfies the conditions of IMF. Write down $c_{1}=h_{j k}$, and $c_{1}$ is the first IMF component of signal $x(t)$. 
4) Decompose $c_{1}$ from $x(t)$ and get:

$$
r_{j}=x(t)-c_{1}
$$

Set $r_{j}$ as the original data to repeat the above procedure to get the second component $c_{2}$ which satisfies IMF conditions. Repeat this circulation so as to get the $\mathrm{n}$ component:

$$
\left.\begin{array}{c}
r_{1}-c_{2}=r_{2} \\
\ldots . . \\
r_{n-1}-c_{n}=r_{n}
\end{array}\right\}
$$

This circulation ends when $r_{n}$ becomes a monotone function and cannot be extracted the component that satisfies the IMF conditions. From (2) and (3), the following equation can be obtained:

$$
x(t)=\sum_{j=1}^{n} c_{j}+r_{n}
$$

In equation (4), $r_{n}$ is residual component and the original data is expressed as the sum of IMF component and a residual component.

Perform the Hilbert transform on the IMF components that obtained by EMD:

$$
\begin{aligned}
& x_{i}(t)=i m f_{i}(t) \\
& y_{i}(t)=P \int_{-\infty}^{+\infty} \frac{X(\tau)}{t-\tau} d \tau
\end{aligned}
$$

In equation (6), $\mathrm{P}$ is the Cauchy primary vector, by transform, $x_{i}(t)$ and $y_{i}(t)$ compose the analytic signal $z_{i}(t)$ :

$$
z_{i}(t)=x_{i}(t)+i y_{i}(t)=a(t) e^{i \theta(t)}
$$

Among the above equation,

$$
\begin{aligned}
& a(t)=\left[x_{i}^{2}(t)+y_{i}^{2}(t)\right]^{1 / 2} \\
& \theta(t)=\arctan \left(\frac{y_{i}(t)}{x_{i}(t)}\right)
\end{aligned}
$$

The instantaneous frequency can be defined as:

$$
k(t)=\frac{d \theta(t)}{d t}
$$

Then the data can be expressed as:

$$
x(t)=\operatorname{Re} \sum_{j=1}^{n} a_{j}(t) \exp \left(i \int k_{j}(t) d t\right)
$$

At present, the residual component $r_{i}(t)$ is neglected, for it is a monotone function or constant value.

The three-dimensional time-frequency spectrum $H(\omega, t)$ of $x(t)$ can be obtained:

$$
H(\omega, t)=\sum_{j=1}^{n} H_{j}(\omega, t)
$$

By time integral of the above equation, get the twodimension marginal spectrum $h(\omega)$ :

$$
h(\omega)=\int_{0}^{T} H(\omega, t) d t
$$

\subsection{IMF signal feature extraction technology based on correlation coefficient}

In view of the fact that the complex components of the roller signal and the de-noised signal still produce extra IMF after EMD decomposition, which is easy to cause misjudgment, an improved HHT method based on correlation coefficient was adopted to extract the effective IMF component. Concrete analysis in the process, according to each IMF component and before the EMD decomposition signal phase relationship value to select the effective component of the IMF, the greater the correlation coefficient, the more valid features of the original signal contained in IMF, the $d_{f}$ for the IMF component and the correlation coefficient of signal before the EMD decomposition, taking $d_{f} \geq 0.1$ for the selection of effective IMF conditions $\left(d_{f}<0.1\right.$, the correlation of signal usually is very small, so the selection of $0.1 \leq d f \leq 1)$. Hilbert spectrum was obtained by Hilbert spectrum analysis of effective IMF. The flow chart of roller fault diagnosis based on semi-soft threshold and improved HHT is shown in Figure 4.

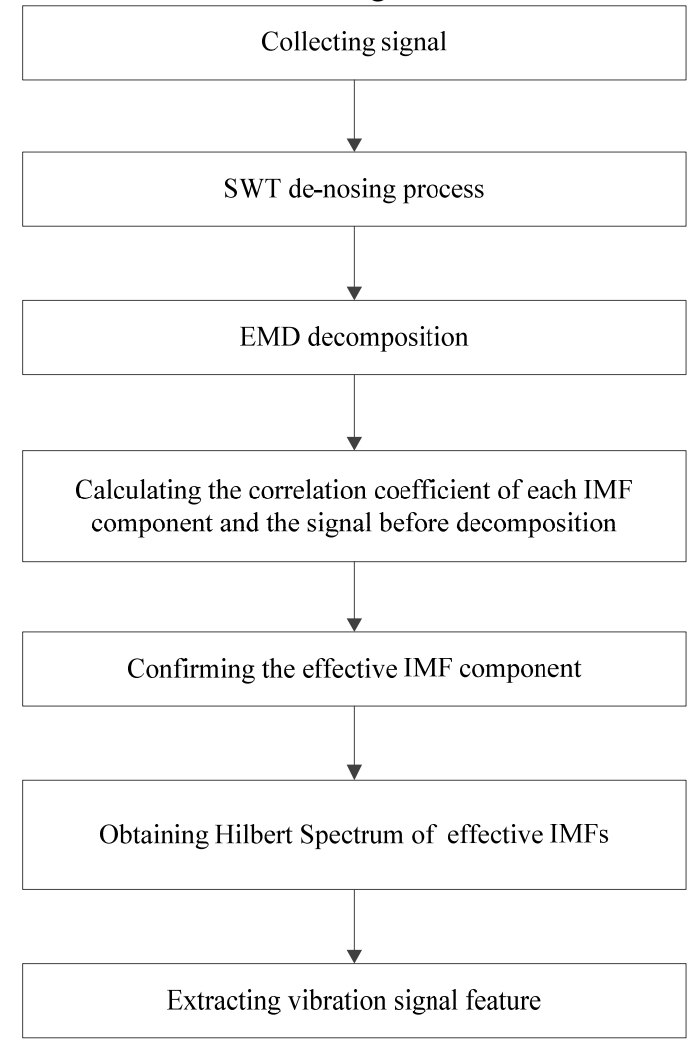

Figure 4. Flow chart of roller fault diagnosis based on wavelet SWT and improved HHT.

\section{CRDM fault feature extraction based on SWT and improved HHT vibration signal}

Figure 5 is the roller original vibration signal a(t) of CRDM life test. After de-nosing of a(t) by the SWT, the decomposing result of EMD is showing in Figure 6: get 15 IMF components and 1 residual component Res. 


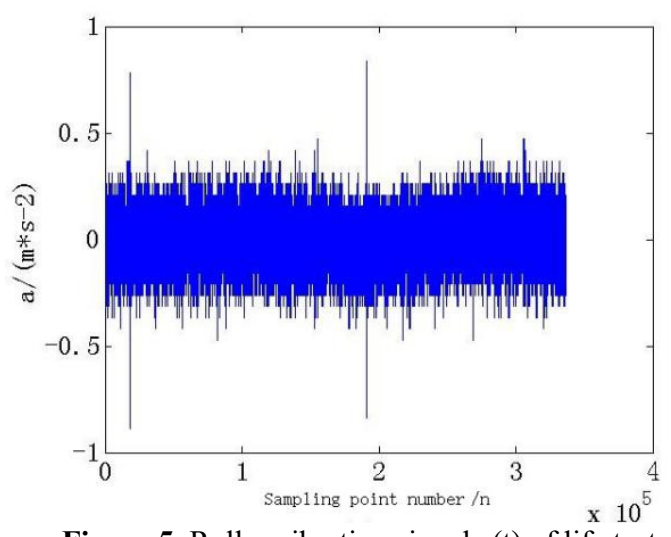

Figure 5. Roller vibration signal a(t) of life test.
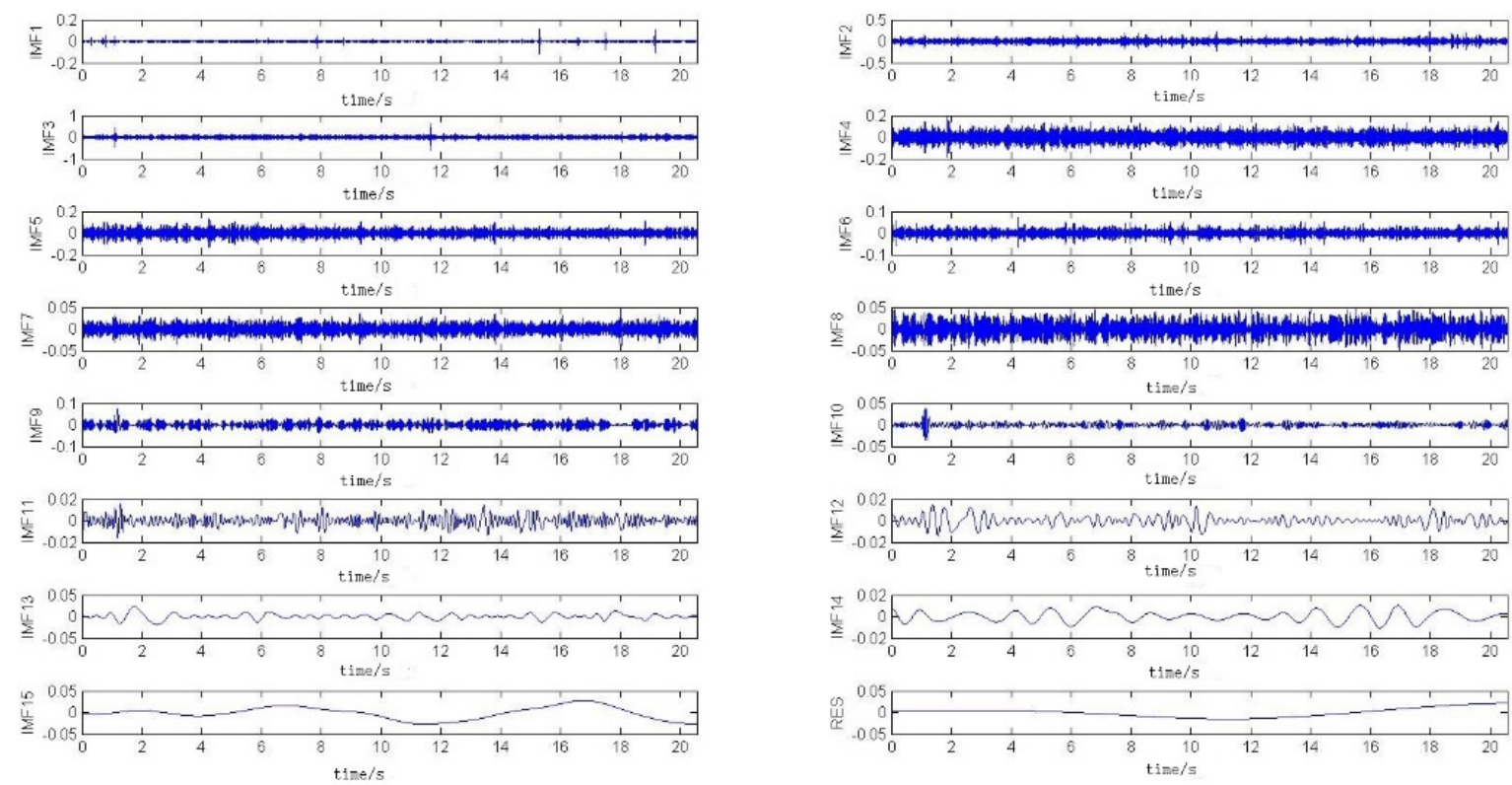

Figure 6. Original signal EMD decomposition results.

For different IMF components include different signal features, the effective IMF component should be selected according to the correlation coefficient size between the original signal $\mathrm{a}(\mathrm{t})$ and each IMF component, that is, the IMF components satisfied the condition of $D x y(f) \geq 0.1$ are selected as effective IMF component and calculate their Hilbert spectrums. Table 1 is the correlation coefficient of IMFs and $a(t)$. It can be known from table 1 that the correlation coefficient of IMF1-IMF9 are greater than 0.1. For IMF1-IMF3 are high-frequency noise components, therefore IMF4-IMF9 are selected as the effective IMF set, and get their Hilbert spectrums.

Table 1. Correlation coefficient of IMFs and a(t)

\begin{tabular}{lllllllll}
\hline IMF & 1 & 2 & 3 & 4 & 5 & 6 & 7 & 8 \\
\hline$D x y(f)$ & 0.5219 & 0.5189 & 0.4705 & 0.2298 & 0.2049 & 0.1377 & 0.1265 & 0.1217 \\
\hline IMF & 9 & 10 & 11 & 12 & 13 & 14 & 15 & \\
\hline$D x y(f)$ & 0.1059 & 0.0925 & 0.0762 & 0.0662 & 0.0548 & 0.0516 & 0.0291 & \\
\hline
\end{tabular}

The roller of CRDM is a low speed rotating part. Given the rational speed is a rpm, the revolution frequency is $\mathrm{a} / 60 \mathrm{~Hz}$. It can be seen from the analysis that the vibration characteristic spectral lines are mainly reflected in the low frequency band. From figure 7 , it can be found that the $\mathrm{a} / 5 \mathrm{~Hz}$ spectral lines of IMF4-9 are relatively obvious, among which the $\mathrm{a} / 30 \mathrm{~Hz}$ spectral lines of IMF4-7 are obvious too. By analysis, the rotor and the roller are in a symmetrical distribution and during the rotating process, the two rollers engaged with the screw at the same time.
Therefore, the two-frequency multiplier a/30Hz spectral line should appear in normal operation. For there are 4 rollers on the rotor, each roller has 3 paralleled gear teeth; when the rotor rotates a turn, the 12 gears of the 4 rollers contact with the screw at the same time, therefore, the obvious twelve-frequency multiplier $\mathrm{a} / 5 \mathrm{~Hz}$ spectral line appears in frequency spectrum. In the EMD decomposition of vibration signal, with the deepening of decomposition levels, the feature information of roller signal gradually decreases, so as the energy, and the 
$\mathrm{a} / 30 \mathrm{~Hz}$ spectral line disappears with them.
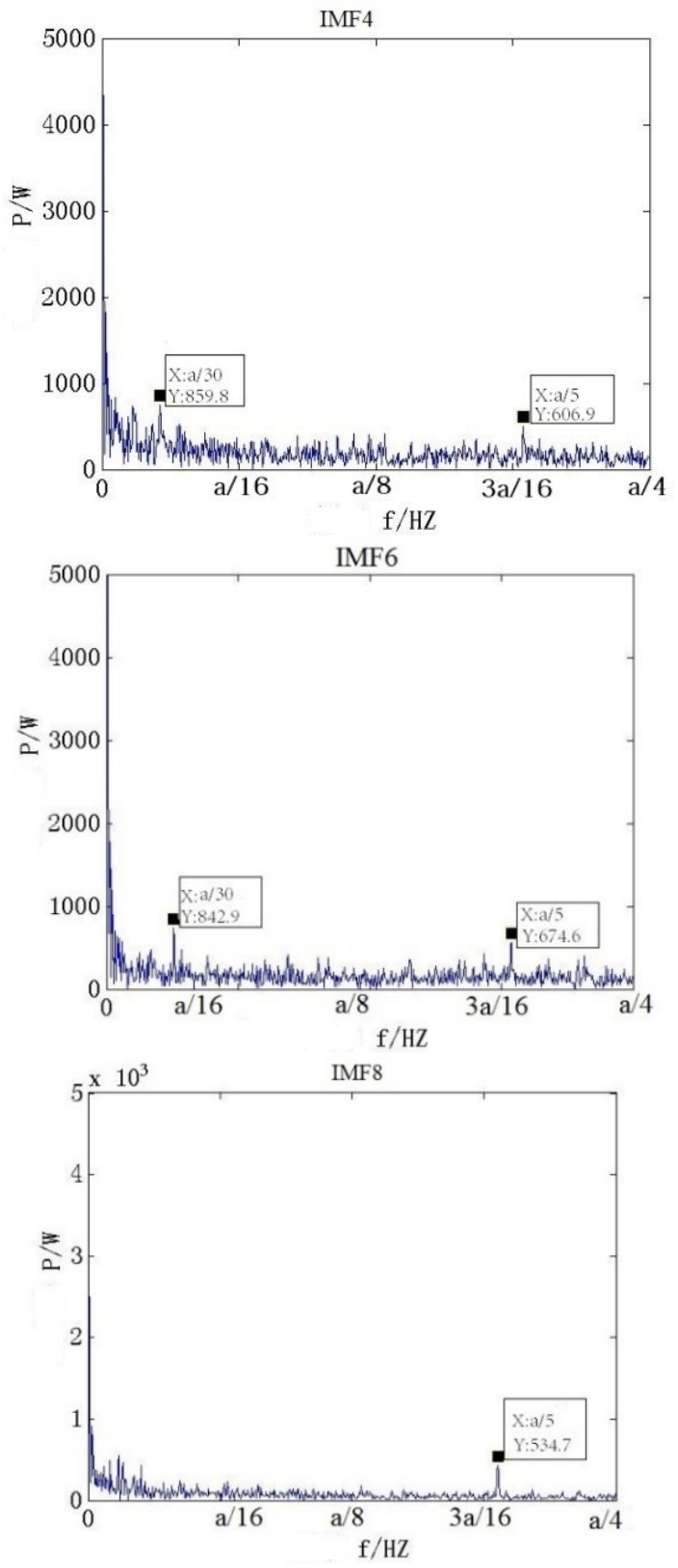
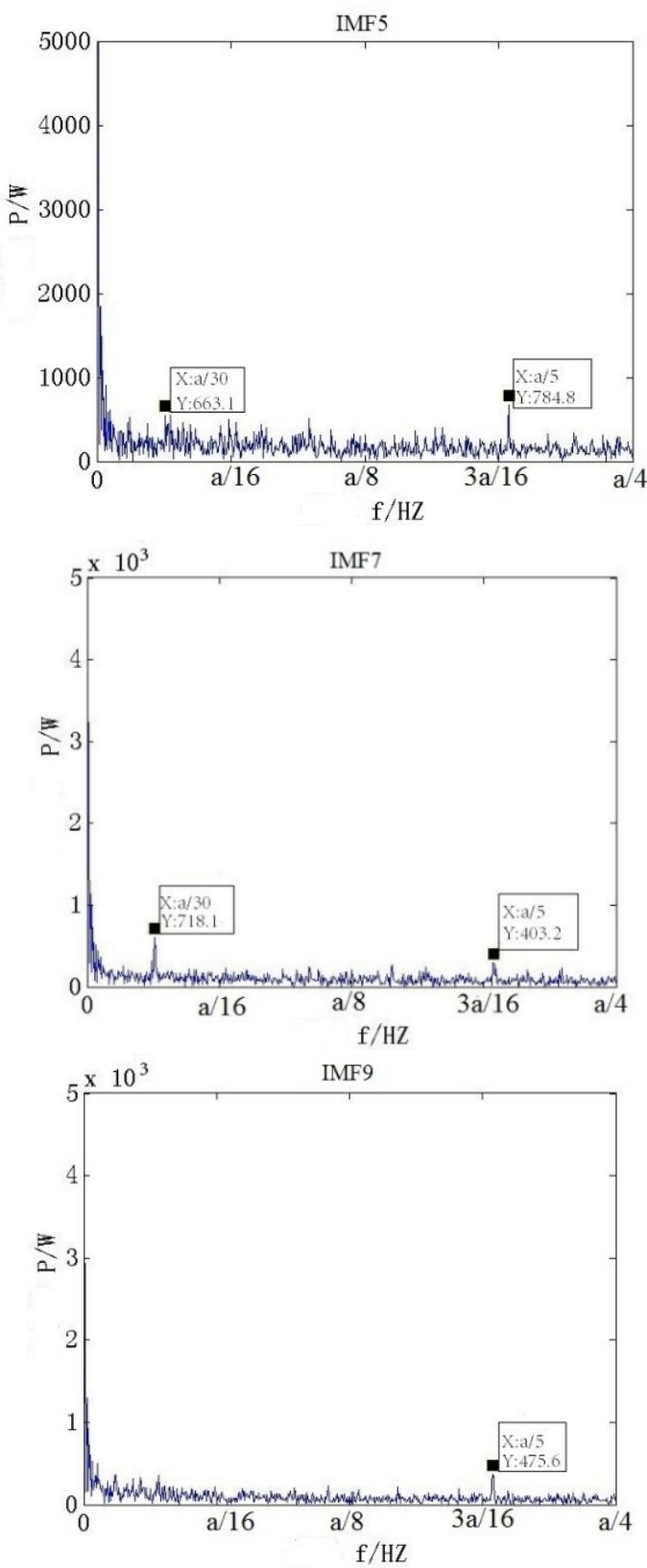

Figure 7. Hilbert spectrum of effective IMF set of the vibration signal from life test.

Select the roller vibration signal of simulated defect test, as shown in figure 8. Compared with the roller vibration signal of life test, the roller vibration signal of defect test is of more energy and more impact composition, as well as regular periodic variation. All these features accord with fault feature of the defect roller and are extracted with the same feature extraction technology. Figure 9 is the EMD decomposition results after SWT process. 


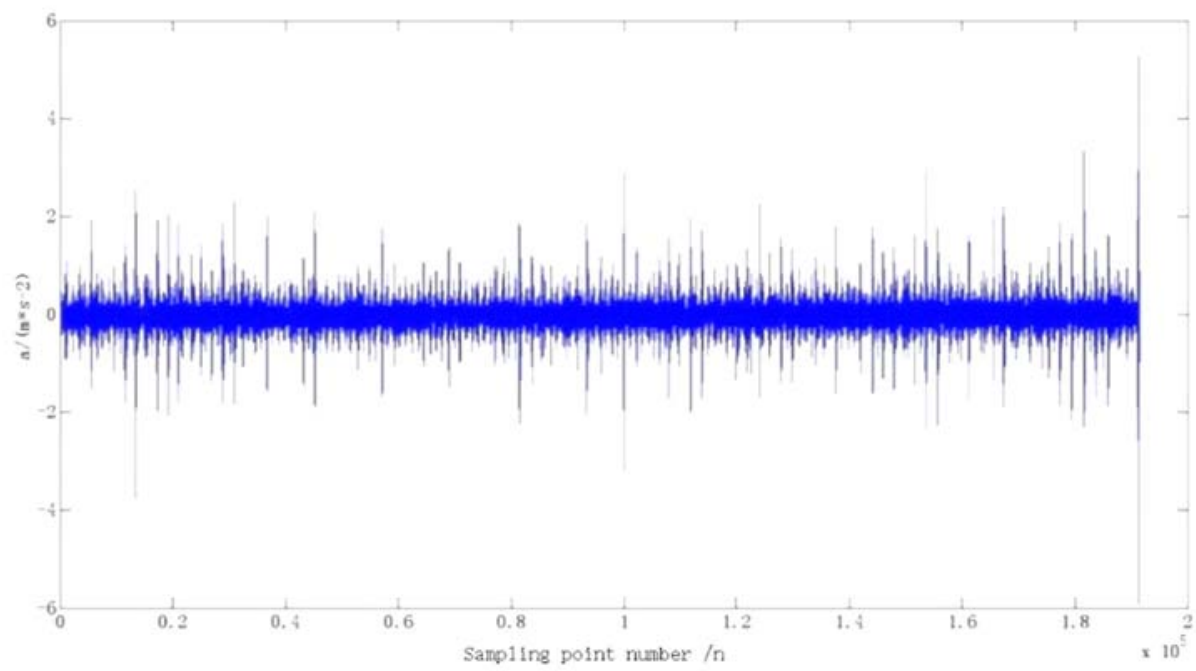

Figure 8. Roller vibration signal $b(t)$ of simulated defect test.
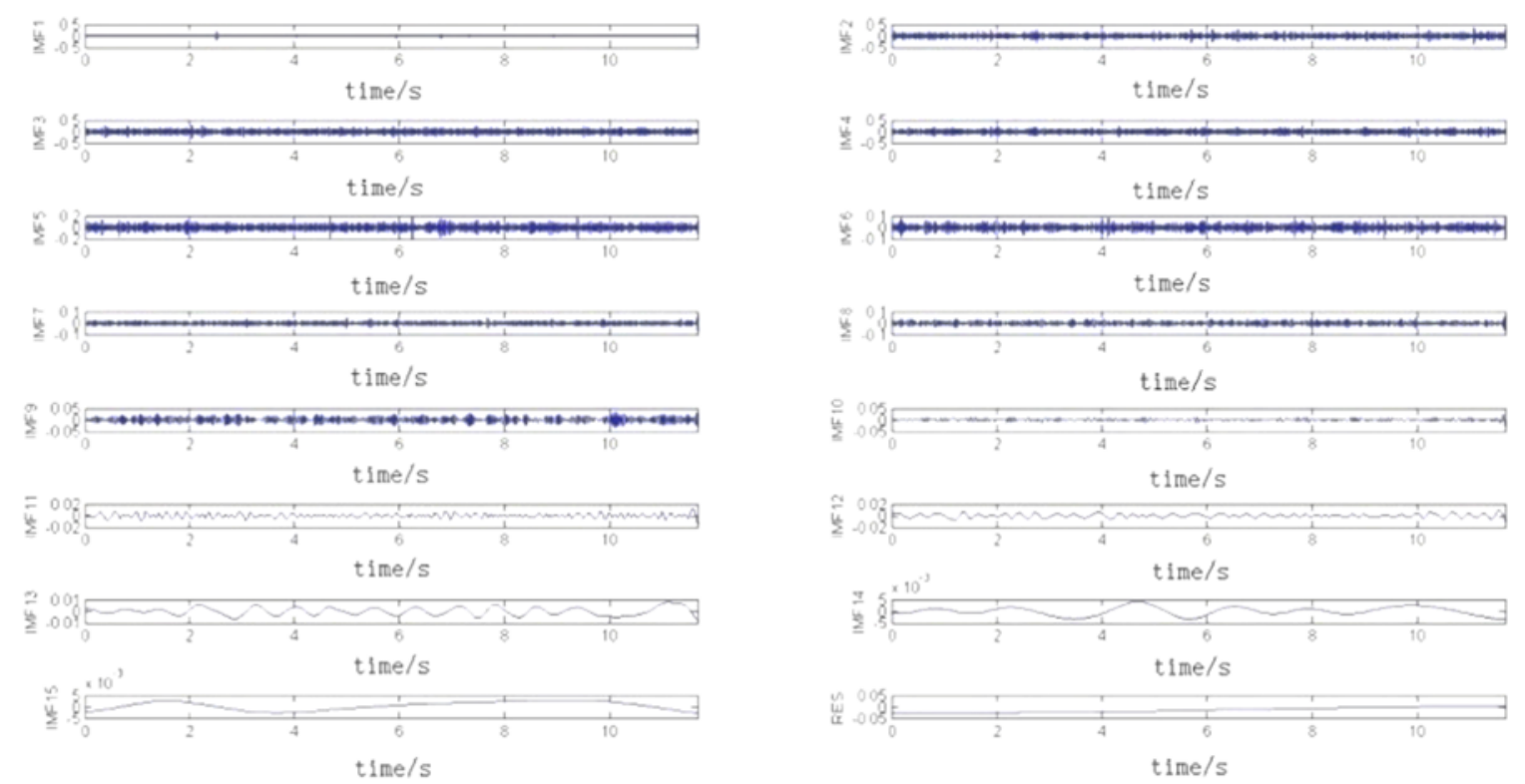

Figure 9. Simulated defect test signal EMD decomposition results.

Table 2 is the correlation coefficient of IMFs and $b(t)$, among which the effective IMF set is IMF4-IMF7, and figure 10 is the Hilbert spectrum. By analysis figure 10, it can be found that $\mathrm{a} / 60 \mathrm{~Hz}$ spectral line appears in the roller vibration signal spectral line of simulated test, for in the simulated test a man-made defect was made on the gear teeth. During the process of the roller and the lead screw engaging, impact is produced when they engage in the defect. Therefore, when the roller rotates once, an impact signal is produced in the defect. The passing frequency of the impact is the rotating frequency. With the increasing of operation time, the energy of impact signal becomes higher, and the amplitude of the vibration signal time domain spectrum becomes bigger.

Table 2. Correlation coefficient of IMFs and $b(t)$

\begin{tabular}{lllllllll}
\hline IMF & 1 & 2 & 3 & 4 & 5 & 6 & 7 & 8 \\
\hline$D x y(f)$ & 0.5724 & 0.5236 & 0.4908 & 0.3054 & 0.1148 & 0.1127 & 0.1076 & 0.0898 \\
\hline IMF & 9 & 10 & 11 & 12 & 13 & 14 & 15 & \\
\hline$D x y(f)$ & 0.0825 & 0.0504 & 0.0443 & 0.0244 & 0.0215 & 0.0197 & 0.0142 \\
\hline
\end{tabular}



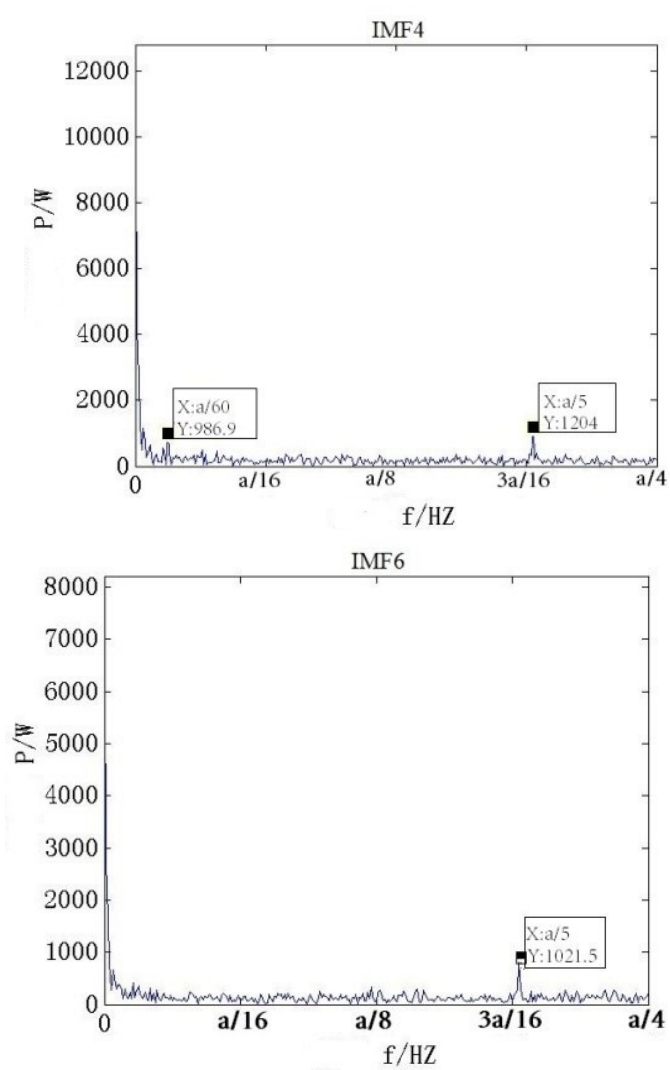

Figure 10. Hilbert spectrum of effective IMF set of the vibration signal from simulated defect test.

\section{Conclusions}

(1) Although the sealed reluctance motor type CRDM vibration signal has many transmission surfaces, nonstationary, nonlinear and easy to be disturbed by noise, etc., reasonable monitoring methods, signal noise reduction and feature extraction methods can be adopted to realize the CRDM roller fault status monitoring and evaluation.

(2) Based on SWT and improved Hilbert method can effective to CRDM vibration signal feature extraction, in this paper, based on SWT and improved Hilbert method from to identify the driving mechanism transition status, in theory, can also be applied to other drive rotor status recognition.

\section{Acknowledgments}

The work described in this paper was supported by grants from the Natural Science Foundation of HuBei, Province(grant 2019cfc889) and the Science and Technology on Reactor System Design Technology Laboratory Operation Foundation of Nuclear Power Institute of China(HT-KFKT-02-2017101).

\section{References}

1. Yu, J.C. (2020) Marine nuclear power technology. Shanghai Jiao Tong University Press, Shanghai.

2. Hu, Y.D., Ren, W.X., Yan, J.Y. (2016) Analysis of Hilbert Low-pass filter and improvement of
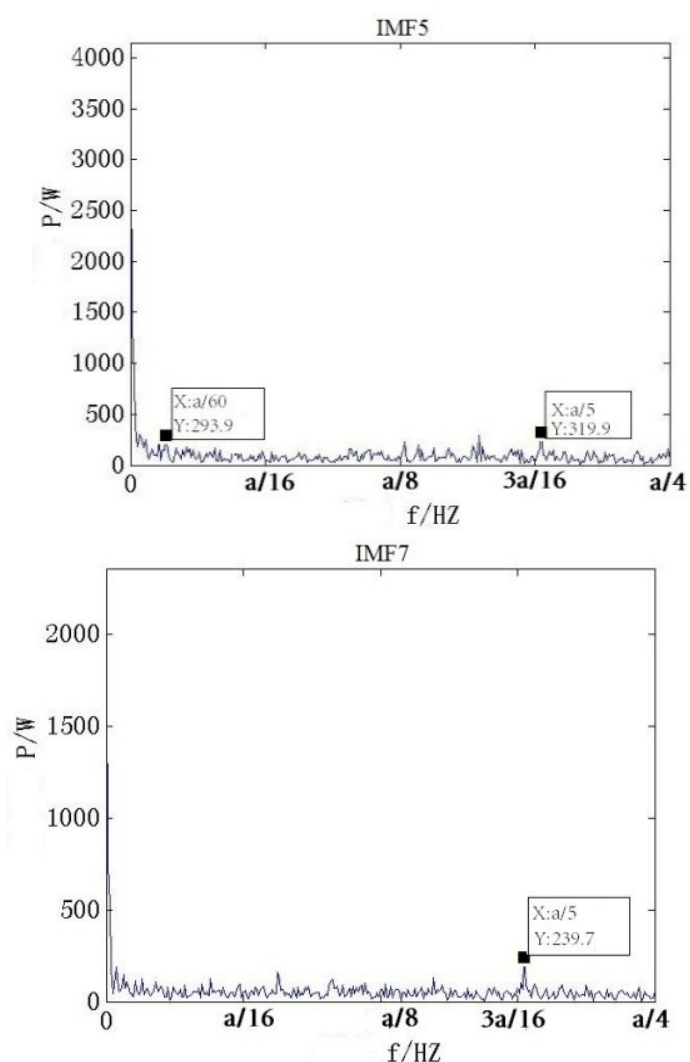

numerical methods. Journal of Vibration, Measurement\& Diagnosis., 36(2): 383-389.

3. Wang, Q.A., Wu, Z.Y., Liu, L. (2016) System recognition based on Hilbert-Huang Transform and ideal Band-pass filter. Journal of Vibration, Measurement\& Diagnosis., 36(6): 1065-1070.

4. Xu, Y.H., Zhao, Y. (2011) Identification of power quality disturbance based on short-time fourier transform and disturbance time orientation by singular value decomposition. Power System Technology., 35(08): 174-180.

5. Kong, L.J. (2014) Matlab wavelet analysis super learning manual. Posts \& Telecom Press, Beijing.

6. Zhang, X.D. (2015) Modern Signal Processing. Tsinghua University Press, Beijing.

7. Cheng, J.S., Yu, D.J., Yang, Y. (2004) Energy operator demodulating approach based on EMD and its application in mechanical fault diagnosis. Chinese Journal of Mechanical Engineering., 40(8): 115-118.

8. Qin, S.R., Zhong, Y.M. (2006) A new envelope algorithm of Hilbert-Huang transform. Mechanical Systems and Signal Processing., 20 (8): 1941-1952.

9. Xiang, L., Yan, X.A. (2014) Performance contrast between LMD and EMD in fault diagnosis of turbine rotors. Journal of Chinese Society of Power Engineering., 34 (12): 945-950.

10. Shi, L., Chen, L., Zhang L.M. (2018) De-noising of vibration signal of sealed magnetoresistive motor CRDM based on semi-soft wavelet threshold and Hilbert transformation. Atomic Energy Science and 
Technology., 52 (2): 314-319. 